Ciência eNatura, Santa Maria, v. 37 Part 1 2015, p. 286-297

\title{
Investigating the affecting factors on quality of life in informal settlements' neighborhoods, with an emphasis on the Islamabad neighborhood in Zanjan
}

\author{
Jalal Kamran* \\ Master of Urban Design and Planning, faculty member of Gachsaran, \\ School of Art and Architecture, Islamic Azad university, Gachsaran, Iran \\ jalalkamran11@yahoo.com
}

\begin{abstract}
The importance of quality of life due to the direct impact on the urban community and system has been the subject of study by experts in various fields. In this regard, urban poor neighborhoods due to Inappropriate quality of life is one of challenges faced by the managers, urban planners, sociologists and other related fields. Urban poor neighborhoods as informal settlements due to the informal nature or considering informal, is the location of the poor which has always been in the improper status of the quality of life's indices (like housing, literacy and education, income, environment, etc.). Assessment and evaluations of the quality of life in informal settlements' neighborhoods are one of the most important studies of the empowerment project of these neighborhoods, which its result can be taken into account in providing sound solutions with regard to the quality of life in the mentioned neighborhoods. This paper examines and assess the quality of life in informal settlements' neighborhoods by using two way of library research (theoretical framework) and Field research (collecting statistical data and questionnaires) on the Islamabad in Zanjan as a Case study and try to offer the cooperative strategies to increase quality life.
\end{abstract}

Keywords: Quality of life, Informal settlements, Evaluation, Islamabad. 


\section{Introduction}

$\mathrm{T}$ oday's Quality of life is one of the most important issues facing the world and is the major issues in the development of social policies which encompass issues such as welfare, health based quality of life, growing and satisfying life, poverty and social exclusion and social coordination, altruism and Partnership (Phillips, 2006).

In today's urban society, especially in Third World countries such as Iran, due to the low attention to the regional development, poor services and supplying social welfare of rural residents, lack of integrated programs in the form of rural development, oil-based economy, the focus of services and facilities in urban centers have always been a factor in forced migration of rural desperate residents (sometime optional) and migration to cities (with the dream of achieving a better life) including the metropolitan areas and the cities inability on giving the service and absorption of the delivered population to the context of the city led to the formation of neighborhoods with the improper quality of life which is called informal settlements.

These areas due to poor infrastructure, lack of services, poor environmental conditions, social, cultural and economic issues and problems (such as low urban culture or non-aligned rural culture with the urban culture, Disempowerment, lack of education, low income, residents economic failure and etc.) have always been in a difficult situation in terms of the quality of urban life which have made the more deeply concept such as urban poverty.

What is important is the process of deterioration of the quality of life in these neighborhoods that made the problem more acute and severe; they come to the conclusion in many of the countries that have faced or are facing the challenge of informal settlements that one of the approaches to enhance the quality of life is using various ways such as education, participation, empowerment with parallel review and synergies of the different management and people living in these neighborhoods.

Zanjan Islamabad neighborhood with a population of 34 thousand people (1390 census) with the nature of informal settlements is often in improper situation in terms of different quality of life indices. This neighborhood has faces problems such as lack of education, improperness of the neighborhood's environmental condition, poor quality of housing, high population density by inadequate and low per capita, the lack of municipal services, The weak economic of residents, poor urban culture, Disempowerment, and stigmatization.

\section{Literature review}

\subsection{Quality of life and urban space}

The Quality and the Quantity are two important issues in every fields and regions. The Quantity consist such matters into its territory which is measurable while the quality consist how the things are. Sometimes the quality can be measured or calculated, although the certainty of it was a little bit weaker than the quantity matters because the quality is not stable over the time and changing constantly (seyed Mirzaee, 89: 1376)

The quantity and the quality has been a twoway interaction that affect each other that they sometimes have a positive impact and sometimes may have a negative impact, So that the increasing number of one thing is not equivalent to increasing the quality of that, even this Increasing math may along with the declining the quality of that things.

If the quantity enhancement is not along with the quality improvement, the increasing is instable which certainly followed by problems. Therefore the distinction between quantitative and qualitative characteristics of the phenomenon at every level of the reality is possible and simultaneous neglecting to two aspects can lead to decisive theoretical and practical errors (Kokabee, 6: 1384). Quality of life, as it is known on its prefix, has considered the quality not the numerical Increasing. The concept of quality of life is so abstract that cannot offer the comprehensive definition for it. The main problem in defining such concepts are rapid changes and evolution over time and place (geography).

The history of the concept of quality of life was return back to the economic growth's opposing action that the report of Rome's club as the Limits of Growth was of the first and most 
organized of this reaction. The main emphasis of the Rome's club was on the environment, but gradually the attention to quality of life was beyond the field of environment and also encompass the social and cultural fields, too.

The Judgments on the definition of the quality of life and its determination by the indicators are very difficult. We can noted the development program of the "Jacksnowill" as an example in the conceptual evolution of the quality of life, that in which the quality of life has been defined as a sense of being better, prosperity, satisfactory results of the external environment (Greenwood, 2001: 6).

Because the definition focuses on the importance of the real feelings of interpersonal relationships, it focused on the external environment and shows the overcoming of the external view on the concept of quality of life. However, the quality of life is of the better living conditions in which balance, coordination, compliance and fair equality or necessary background for a life were raised along with the health, security, comfort, peace, happiness, morality and beauty. (Haji-Yousefi, 15: 1381)

So the quality of life is a multi-aspect, partial concepts which has affected by the time, place and individual values. The quality of life is something that is influenced of values. In fact the quality of life is defined according to the individual, collective and national values, definition. (Kharazmi, 10: 1382)

The attention of the Geography to the quality of life's index and its relevance concept is came back to the 1960s decade. David Smith was the first geographer who talk about quality of life, welfare and social justice in geography. He said in investigating the quality of life this question arises that who earns what and where? Although observing people living in different places and in different ways do not have moral implications, but the judgment on the quality of life is inevitably poses on questions about the good or bad, better or worse (Smith, 171: 1381.

This geographer uses subjective social and objective comparison indices to assess quality of life, welfare and social equity that questionnaire is used in order to measure subjective indicator and observation and statistics is used for the objective one. Smith emphasized indices encompasses of health, housing, public services, , family happiness, education, employment opportunities, salaries and wages, food, the right, the life expectancy, consumption's per capita of animal protein, the percentage of school enrollment, the average number of telephone and newspapers and so on up (Smith, 160: 1381).

In the 70s, David Harvey also published four paper about this issues. Food, shelter, health care, education, social services and environmental services, consumer goods, recreational facilities, neighborhood's pleasant and transportation devices are nine categories of human needs that according to Harvey people needs the least of it.

The second emphasized point by Harvey is in helping the public interest. Allocation of resources to one area affects other areas. In fact, it can re-organize the current Coefficients of regional cumulative by the foundation of a new space systems or adjust the previous adverse effect by affecting on the current allocation. According to Harvey, allocating more resources to the region is acceptable when the aim is to overcome the environmental problems (Harvey, 1376: 49-77).

Harvey, in justice, natural and geographical disparities book (1996) pays on factors such as income, different spaces of life, race and etc. and their effects on poisoning, low blood safety, health care and .... (Harvey, 1996: 394)

Also a book as the space and social inequality written by Emad Afroogh that he has been trying to evaluate and assess the effect of space on people's behavior through the measuring of the level of household head's education, per capita income, per capita infrastructure, housing ownership status and job status and to determine

Many tool has been developed to assess quality of life in different societies. The economists, due to having a powerful tool to measure, based on their assumptions, were the first who indirectly paid this issue. Gross domestic product (GDP) was the first indicator that economists was compiled based on the following assumptions for measuring social progress and implicitly the quality of life.

The human is a creature who lives with economic logic. Economic logic dictates that a man to be competitive and maximize. If favorable economic conditions is emerged, the quality of life is improved by itself. As the 
economy flourished and developed the basic human needs will be provided.

Critics say that neither of these assumptions is true and not the certain means of the economic development led to improve the quality of the people's life. People in developed countries have achieved economic prosperity, but do not feel their lives are of good quality. So GDP growth, which is a measure of economic growth, cannot be considered as an improvement criteria of the quality of the people's life.

In order to solve this problem the other indices were developed that also covered social factors. GPI with 18 variables is one of this indicators. This indicator has tried to compensate the economic perspective weakness by adding variables such as unemployment and part-time jobs to death in the accident and pollution and military expenditures.

FISH index was developed in 1970 and has greater attention to social health by 16 variables. Or HDI which is the Human Development Index and tries to give attention to all aspects of quality of life such as socio-political dimension by a comprehensive look. The human development index was designed by "mahbob alhagh" in 1975 and since that year, the United Nations Development Program (UNDP) assess the situation of the countries every year and rank them. Also there are indicators for assessing the quality of urban life in the US, Canada and European cities.

However, the Institute of EIU also has developed an index by the name of Quality of Life Index, which in 2015, evaluated and ranked 111 countries including Iran based on 9 following variables that are most of these are of objective conditions.

Material prosperity which is measured with per capita GDP and PPP (power, parity, price), life expectancy in years, security and political stability, family life; divorce rate per 1,000 inhabitants, social life; the presence of social circles and organizations and religious places, weather and geography; the favorable climate in terms of heat and cold, job security; unemployment, civil and political liberties, gender inequality; the ratio of women's average income to men's.

In the EIU ranking in 2015, Iran is obtained the rank of 88 among 111 countries in terms of quality of life on the ninth variables.
Listed indicators, don't pay attention to the subjective aspect of quality of life. However, the Institute of nef (the new economics foundation), which is a non-profit organization and active in the field of quality of life's improvement, had developed an index that called the happy planet index that in which a combination of subjective and objective variables were measured. In 2005 report of this institute, a model had presented that in which the process of resources conversion to quality of life was defined as: planetary resources as inputs should convert to outputs through ten tools that is nothing but long life and happy life for the people. This ten instruments as follows: 1- Government and rule 2- the local community (urban management) 3Technology 4- Health 5- Education 6- family 7values 8- economy 9- Employment 10consumption.

Consequently it must be admitted that the assessment and measurement of quality of life depends on the benefiting level of community and resident's subjective feeling on living's environment quality of life. Resident's subjective feeling of living space depends on the amount of available services, resident's services and facilities' needs, mental and social well-being, the needs of new requirements and many other factors.

David Harvey, in social justice and the city book, introduce needs as a relative concept and does not consider humans needs as a fixed factors. Because meeting the needs over the time cause to produce a new requirement that access to it, will be considered the quality of life indicators and its determination (Harvey, 1376: 106-101).

In urban communities, what is known as Quality of urban life's index, is meeting the needs of residents in various aspects such as socio - cultural, economic and environmental, etc., as well as the amount of access and providing needs.

Urban residents have different criteria for the quality of urban life, for example in the social dimension, the solidarity and attachment to a place in which people live together may be a criteria of the quality of life. They use personal solidarity in this common life and by saying my town, my street and my community and so on ..., they speak in such a way that we can feel social capital. Therefore if we want to raise the 
city's quality of life requirements, they are as follows: in the social dimension (social justice), in the economic dimension (economic viability) and in the environmental dimension (viability or possibility of life) (sarafi, 1380: 7).

\subsection{Informal settlements and quality of life}

In recent decade's self-grown settlements within or adjacent urban area, with an unpleasant scape, out of urban planning and without any building permission, have grown rapidly and they are in a physical break from the city in spite of the Function's coherence with the main town. Accumulation of people, mainly low-income and poverty-stricken immigrants is created an environment with very low quantity and quality of life (especially in comparison to the city), that the residents of these settlements been in the risk of the social, natural and environmental impairment and has caused their separation from the main city. Informal settlements are not a transient and vanished phenomenon and are growing strongly in the developing countries. Thus, the necessity of rapid improvement of the situation has noted in the most important international documents such as the Millennium Development Goals (Sarafi, 33: 1383).

As mentioned, the informal stigma are inevitably attached to their residents in many cases due to the informal nature of these settlements, they are not always in a good conditions in terms of quality of life (housing, health, environment, income, public services, social security and ...) and this reason causes the creation of all sorts of multiple problems in the city and urban system.

\section{Methodology}

This paper tries to investigate some of the Islamabad neighborhood's quality of life index by using library, documentary and field studies (questionnaire (240 samples)) and then mention to the role of cooperative strategies and the use of participatory methods in order to enhance the neighborhood's quality of life. So it is assumed that the use of Potential capacities or local ignored capacities (such as social capital and participation) can be effective in improving quality of life.

\section{Case Study}

\subsection{Islamabad and quality of urban life}

Islamabad neighborhood with a population of 34 thousand people was formed as one of the marginal neighborhoods of the city of Zanjan in1350s. In spite of the fact that this neighborhood having designed structure, due to not being located in the city legal limits of the 1355 zanjan master plan, it was exploited by Dealers and land-grabber despite 30 percent construction and was sold out to the rural migrant population.Today the rural is of the informal settlements of the zanjan city, because of Unauthorized and non-standard constructions. Different techniques and methods such as data collection and available statistical data, layout and executed plans in the city and neighborhoods (including detailed plan), the calculation of the quality of life of the base map, questionnaires and interviews (240 samples) were used in order to check the quality of life in this neighborhood.

In Overall, three main indices (physical and environmental, socio- cultural, economic) were used to measure the quality of life and numerous sub-indices were considered for each of the main indicators.

\subsection{Physical and environmental indicators of the neighborhood}

Available statistics, base maps, field observations, local look, questionnaire were used to measure this index and the results of which are as follows.

\subsection{Per capita:}

Housing units: residential units per capita is of 15 square meters per person, while this per capita is of 27 square meters in Zanjan and the minimum standard of the residential units in Iran is 30 square meters .

Green space: green space per capita is of 0.76 square meters per person, while the minimum standards in the neighborhood level is of 1.5 square meters.

Healthcare: the healthcare per capita is of 0.03 square meters and its standard is of 1.52 square meters per person.

Education: the education per capita is of 0.81 square meters per person while its standard in the neighborhood level is of 4.4 square meters. 
Numbers of rooms: There is 2.1 room in this neighborhood per household that according to neighborhood household size (4.2), the per capita is 0.5 , room per person which shows the improper situation.

Parceling: Based on derived calculations from base map, the area of the residential parcel in the neighborhood is of 86 square meters while the average area of residential land use in the city of zanjan is of 170 meters.

Also, the average of the residential floor area (building) in the neighborhood is of 52 square meters while this is despite the fact that nearly 70 percent of the residential parcels (arena) are under 100 meters and approximately 20 percent are under 50 meters. Because of household size and population density, it must be said that the quality of life is not in proper situation from this point of view.

Quality of housing: In terms of building structures, 88.4 percent of the neighborhood's residential units have the mixture of iron, brick and wood with unstable connection that cannot be resisted in times of natural disasters.

Facade materials: 72 percent of the neighborhood's residential units have an exterior view of the cement (black cement), which have created improper visual quality for residents.

Building age: about 89 percent of the neighborhood's residential units have more than 20 years old that shows high age according to the building's quality and skeleton.

Building's sun exposure: 40 percent of the neighborhood's residential units have totally inappropriate situation of using sunlight and sun exposure and 51 percent have relative sun exposure. Because the sunlight is one of the basic and natural human body's needs, so houses nonexposure to sun light have created problems for the residents.

Neighborhood's urban Facilities and services:

Based on completed questionnaire (240 samples) the following results have achieved in terms of access to urban facilities and services:

Facilities and health Services: The state of access to this index have considered inappropriate by nearly 48 percent of respondents.

Recreational and sports facilities: Neighborhood's sports and recreational facilities have considered inappropriate by 78 percent with respect to the financial conditions and economic power.

Sewage disposal: Neighborhood's sewage disposal system are in the form of passages disposal and absorbed well and domestic sewage are flowing in the streets. The state of neighborhood's Sewage disposal are considered improper by about 57 percent of residents.

Waste disposal: 41 percent of the population considered that the waste disposal situation is unfavorable. It is worth noting that due to the lack of urban culture, the problem of Waste disposal is one of the health problem in the neighborhood.

The alleys and streets state (coverage and quality): According to 64 percent saying of residents, the neighborhood's alleys and streets state is considered unfavorable.

Access to public transport: According to neighborhood's checkered grid and ease of public transport's traffic within the neighborhood, this index have been in optimized situation in comparison with other indicators, So that the only 8 percent of the population considered the state of access to public transportation as unfavorable. Thus, this index can be the neighborhood's strengths points.

Educational facilities: in opinion of 51 percent of the population, the state and quality of the neighborhood's training centers is in improper conditions.

Green space: 55 percent of the population have chosen a bad or very bad situation in the point of access to green space and its sufficiency. However, due to the construction of a new park in Islamabad, this index is improving.

According to the given data and information, we can conclude that this neighborhood is not been in a favorable position and state in the environmental and physical dimension and always is in a lower rank in terms of quality of life in expressed index.

\subsection{Economic Indicators}

In order to investigate this index, which always had and have direct and indirect impact on the other indicators, the following subindicators are used: 
Occupation: The rural migrants are inevitably turned to Manual labor and occupation because of a variety of reasons such as lack of education, lack of knowledge of the city, lack of knowledge and expertise with urban jobs and they earn their family livelihood in this way. In fact, the Islamabad neighborhood can be known as workers center of the Zanjan city.

Information and sampling conducted by the University of Medical Sciences on married population aged 15 to 64 years and the employment of married women aged 15 years have been used In order to check the job index.

Thus, of the 967 chosen samples of the neighborhood 10.4 percent of the active population aged 15 to 64 years were married, 70 percent were workers, 9 percent were the employee, 3 percent were teachers, 1.1 percent were military and 8.9 percent were shopkeeper. So most of people have workers jobs with relatively low-income.

The amount of income: Based on the taken Sampling of the 240 samples, the average income of the family is 200 thousand tomans per month that according to the declaration of the Statistical Center of Iran in 1387 the poverty line in Iran have been announced as of 480 thousand tomans for a5 people family.

Therefore, according to neighborhood's household size means 4.2 and generalization of the declared poverty line to household size, any other family of the neighborhood must have of 380 thousand tomans per month in order to reach the determined poverty line.
So this neighborhood is in improper and unfavorable situation in terms of income power that this factor has been a reason for the declination of the quality of life on other indicators such as housing quality.

Adequacy of living income: based on the taken Sampling, 59 percent of the households have mentioned their lack of income Adequacy on living expenses, 58 percent mentioned their relative Adequacy and only 5 percent of households have mentioned their income's Adequacy on living expenses. It must be said that most of the household spending has been devoted to food, clothing, housing and household affairs as the relevant complications, because of the low expectations and satisfying of the many families. Another point is that in many cases because of economic weakness in supplying the noted needs (housing, clothing and food) the mentioned factors are also inappropriate or so called cheap.

For example, based on the Sampling of the 997 households of the Zanjan University of Medical Sciences, products such as olive oil, cream, animal oil are used once a week by only 0.05 percent of the households and honey are used once a week in their diet and meal Plan by only 14 percent of people. This data suggests that the nutrition in the household are going toward the cheap food.

The assets of the family in the housing units: the level of having the life's facilities and furniture is another indicators in evaluating the quality of life. Given that this index is directly related to economic indicators, so, it is considered as the economic indicators in this article.

Table 1: the Asset and having's levels of families of home furniture

\begin{tabular}{|c|c|c|c|c|c|c|c|c|c|c|c|c|c|}
\hline 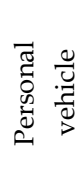 & 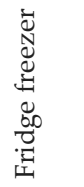 & 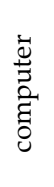 & 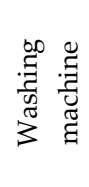 & 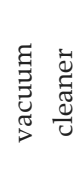 & 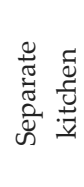 & 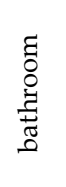 & 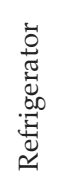 & 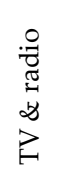 & $\begin{array}{l}\frac{0}{0} \\
\frac{0}{2} \\
\frac{0}{2} \\
\frac{1}{1}\end{array}$ & 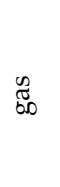 & 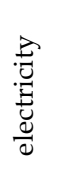 & 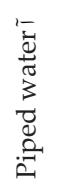 & \\
\hline 10 & 5 & 20 & 35 & 75 & 76 & 86 & 96 & 94 & 95 & 100 & 100 & 100 & $\begin{array}{c}\text { The level } \\
\text { having }\end{array}$ \\
\hline
\end{tabular}


As the above table shows, having level of households of the primary necessities such as electricity, water, gas are in an optimal condition, but in having the secondary needs such as computers, washing machines, refrigerators and personal cars are not in a good condition.

This suggests that the economic weakness of the residents had been always a factor in the level of enjoyment of the needs and the available furniture in the house. What can be concluded of the expression of the economic Index is that the economic weakness of the neighborhood's residents is always a reason on the declination of quality of life in many cases.

\subsection{Demographic, social and cultural Indices}

Available documents and statistics and questionnaire analysis were used to measure this indicator, which its results are as follows:

Neighborhood's household size was 4.2, while this factor is 3.8 in the city of Zanjan. The neighborhood's population Gross density was of 400 to 600 people per hectare and the population net density was 680 people per hectare, while the city's population density is of 200 to 400 people per hectare.

Because the neighborhoods is facing the low or lack per capita, housing units' small parcel as mentioned in the previous paragraphs, this population density is created many social problems such as moral density and psychological pressures of high population density.

\section{Literacy:}

The literacy rate in the neighborhood is of 74 percent that only 37 percent of people have the education higher than the primary school level and only 2.2 percent have a college education. Literacy rates is 56 percent among married couples and also only 17 percent of married people have higher education than the primary school level (conducted sample of 1995 people by University of Medical Sciences) and the literacy rates is 65 percent on married men that only 20 percent of them have had the higher education than the primary school and also the literacy rate of married women is 47 percent that only 13 percent of them have higher education than primary school.
So it can be concluded that the literacy rates in the neighborhood was low which this factor is caused problems such as lack of using printed media (newspapers and Book), un-awareness, problems related to child rearing, reduction of the urban culture, etc..

Leisure time:

According to a completed questionnaire 50.5 percent of the residents devoted their leisure time on watching television or listening to the radio and only 3 percent of the people have visited the entertainment venues and outside of the city in order to spend their leisure time. Its reason has known as being Busy and not having enough time by the neighborhood's people. It is regarded as one of the quality of life indicators, because the entertainment and veering is one of the basic requirements of providing spirit's recreation and rehabilitation, create joy and happiness and the treatment of stress and psychological pressure of urban life and has an important role to increase the quality of life. Therefore, we have observed the low quality of life in this index, due to low veering and recreation in the Islamabad neighborhood.

\section{Criminality:}

According to research In point of criminality rate the Islamabad neighborhood has been ranked in the first place among other neighborhood of the city in crimes such as smuggling, property buyer, rrobbery of the state property and murder. The Islamabad city is in the third place in drug trafficking, second place in consuming drug and selling prohibited goods like alcoholic beverage (Tavakoli, 114) that certainly many cases is of the neighborhood poverty-stricken that is a factor in declination of the quality of life in the neighborhood.

Also, according to completed questionnaires of the households the following results were obtained of social problems in the neighborhood:

As the above table shows, having level of households of the primary necessities such as electricity, water, gas are in an optimal condition, but in having the secondary needs such as computers, washing machines, refrigerators and personal cars are not in a good condition. 
This suggests that the economic weakness of the residents had been always a factor in the level of enjoyment of the needs and the available furniture in the house. What can be concluded of the expression of the economic Index is that the economic weakness of the neighborhood's residents is always a reason on the declination of quality of life in many cases.

4.4. Demographic, social and cultural Indices

Available documents and statistics and questionnaire analysis were used to measure this indicator, which its results are as follows:

Neighborhood's household size was 4.2, while this factor is 3.8 in the city of Zanjan. The neighborhood's population Gross density was of 400 to 600 people per hectare and the population net density was 680 people per hectare, while the city's population density is of 200 to 400 people per hectare.

Because the neighborhoods is facing the low or lack per capita, housing units' small parcel as mentioned in the previous paragraphs, this population density is created many social problems such as moral density and psychological pressures of high population density.

Literacy:

The literacy rate in the neighborhood is of 74 percent that only 37 percent of people have the education higher than the primary school level and only 2.2 percent have a college education. Literacy rates is 56 percent among married couples and also only 17 percent of married people have higher education than the primary school level (conducted sample of 1995 people by University of Medical Sciences) and the literacy rates is 65 percent on married men that only 20 percent of them have had the higher education than the primary school and also the literacy rate of married women is 47 percent that only 13 percent of them have higher education than primary school.
So it can be concluded that the literacy rates in the neighborhood was low which this factor is caused problems such as lack of using printed media (newspapers and Book), un-awareness, problems related to child rearing, reduction of the urban culture, etc.

Leisure time:

According to a completed questionnaire 50.5 percent of the residents devoted their leisure time on watching television or listening to the radio and only 3 percent of the people have visited the entertainment venues and outside of the city in order to spend their leisure time. Its reason has known as being Busy and not having enough time by the neighborhood's people. It is regarded as one of the quality of life indicators, because the entertainment and veering is one of the basic requirements of providing spirit's recreation and rehabilitation, create joy and happiness and the treatment of stress and psychological pressure of urban life and has an important role to increase the quality of life. Therefore, we have observed the low quality of life in this index, due to low veering and recreation in the Islamabad neighborhood.

\section{Criminality:}

According to research In point of criminality rate the Islamabad neighborhood has been ranked in the first place among other neighborhood of the city in crimes such as smuggling, property buyer, rrobbery of the state property and murder. The Islamabad city is in the third place in drug trafficking, second place in consuming drug and selling prohibited goods like alcoholic beverage (Tavakoli, 114) that certainly many cases is of the neighborhood poverty-stricken that is a factor in declination of the quality of life in the neighborhood.

Also, according to completed questionnaires of the households the following results were obtained of social problems in the neighborhood:

Table 2: neighborhood social problems

\begin{tabular}{|c|c|c|c|c|}
\hline Total (percent) & Don't exist & $\begin{array}{c}\text { Partially exist } \\
\text { (percent) }\end{array}$ & Exist (percent) & Social problems \\
\hline 100 & 5 & 25 & 70 & The presence of drug addicts and drug smugglers \\
\hline 100 & 15 & 31 & 54 & The beggars \\
\hline 100 & 10 & 40 & 49 & Fights and conflicts \\
\hline 100 & 16 & 40 & 43 & people's Security at night \\
\hline 100 & 28 & 35 & 36 & Disturbance \\
\hline 100 & 33 & 34 & 32 & Robbery \\
\hline 100 & 36 & 35 & 28 & Lewdness \\
\hline
\end{tabular}

Source: The field research by author 
As the above table indicates, the presence of drug addicts and the beggars were in the first place that matches with the present research. What can be concluded from this table are the existence of social problems in the neighborhood that have a direct impact on the reduction of social security and neighborhood's resident's quality of life.

Having the Social Security services:

Based on the completed questionnaires 70 percent of the population don't have any types of social security insurance (health, retirement, disability, accidents, etc.) that 89 percent of people have been cited that it is due to economic weakness.

Today, because of the importance of the Social Security insurance in creating resident's welfare and comforts, having this factors are of the necessities needs of life and quality of life. Therefore we must be said that, the Social Security policies of the government (as the most important task of the government) does not apply to these dear people and the neighborhood's quality of the life is reduced.

Of course, it must be said that most of the supporting organizations clients such as Social Welfare Bureau and Charity Committee in zanjan city is of this neighborhoods, so that, according to obtained statistics of the city first zone's charity committee, it is obvious that 2,400 $(45 \%)$ people are the resident of Islamabad of 6 million clients of Zanjan. But these organization's support are limited to providing health care insurance or monthly annuities that most of people do not use them because of their self-dignity.

\section{Assessing the prioritization of the neighborhood's resident's needs in order to increase the quality of life}

In the provided questionnaire that the heads of households was addressed, the household's heads were asked to assess their overall situation of their family life. The answer are simply but significantly defined in five choice as very bad, bad, normal, good and very well. The economic situation of 4.8 percent of the respondent's families is considered as good and very good. 54 percent checked the normal status and 41.2 percent of the respondents checked the bad and the very bad choice for their family's economic situation.
Then the people who checked the bad and very bad choice to define their general life's condition were asked to mutually compared the six different items depending on its importance relative to other items in order to achieve better life's conditions. The six items are adequate housing, employment, insurance, education, urban facilities (such as parks, hospitals, libraries, public transportation, etc.) and financial support of the government. These options are based on the definition of poverty based on different theories.

Geographers are more interested in items such as the adequate housing and urban facilities and these items are is mostly related to the physical quality of urban life. Its solution is urban rehabilitation and renewal. Insurance and jobs Items have an economic look to quality of life and economists pay more attention to these. Government's financial support with a Supportive look to government and literacy item, intends to cultural aspect and its solution is economic and cultural empowerment.

The purpose of bringing these options is to determine the subjective perception of the nature, amount and significance level of different items in the quality of life of those who knew the situation of their family life at low levels,

This section contains 30 questions which are 6 items that have been mentioned previously. These items are compared two by two. In this way that first questioned that which item is more important for your family A or B, then it was asked to say the importance of each item in relation to others. The obtained answer has been assessed by using AHP models (Analytical Hierarchy Process ) that consequently the weighting is as follows.

Table 3- the weight of the determined criteria for prioritization the neighborhood's resident's needs in order to increase the quality of life

\begin{tabular}{|c|c|}
\hline index & weight \\
\hline $\begin{array}{c}\text { The coefficient importance } \\
\text { of occupation }\end{array}$ & 0.35 \\
\hline $\begin{array}{c}\text { The coefficient importance } \\
\text { of adequate housing }\end{array}$ & 0.26 \\
\hline $\begin{array}{c}\text { The coefficient importance } \\
\text { of insurance }\end{array}$ & 0.19 \\
\hline
\end{tabular}




\begin{tabular}{|c|c|}
\hline $\begin{array}{c}\text { The coefficient importance } \\
\text { of literacy }\end{array}$ & 0.11 \\
\hline $\begin{array}{c}\text { The coefficient importance } \\
\text { of government financial } \\
\text { support }\end{array}$ & 0.05 \\
\hline $\begin{array}{c}\text { The coefficient importance } \\
\text { of urban facilities }\end{array}$ & 0.01 \\
\hline
\end{tabular}

As it is specified in the above table, the occupation's item is in the first row by the coefficient importance of 0.35 and the government's financial support and urban facilities are at the end of the table by the coefficient importance of 0.01 . Consequently we can say that in the neighborhood's people's opinion, having a job that will increase the economic potential and power is the most important factor in quality of life.

In the summary of all expressed indicators we reach to this conclusion that the neighborhood of Islamabad is not in a favorable situation in terms of quality of life and will require the intervention necessity to improve the quality of life. Considering that technocratic and monographic intervention by government and managerial agencies are always causing new issues and problems in these neighborhood. Therefore the best way of intervention is using the both cooperative and synergistic approaches among people and different management organizations.

\section{Conclusion}

The quality of life is the historical product of cultural, social, economic and political structural systems and cannot be analyzed separate from the above structures. The Competency of the individual life which meets their basic expectations can be interpreted from the quality of life by second reference to the concept of quality. In fact, the "quality of life" is the today's social's development indicator, which includes the most important factors that determine the living conditions in the community and their personal welfare.
Quality of life is the most basic issues of economic and political science in which economic development's material parameters and domestic production are examined along with the non-financial (material) parameters such as quality of work, level of literacy and culture, health and medical standards, quality of leisure and recreation, environmental conditions, political atmosphere, individual happiness and even liberty and national unity.

Meanwhile, the urban poor neighborhood like informal settlements and neighborhood of the city's margin, as its name "margin" implies are the neighborhoods that are far away from the urban context or at the other view the urban context has driven them to the social, economic, political, and physical margins. Today by posing new concepts such as the quality of urban life, it is imperative to measure the quality of life in the margins and proper solutions has to be found in order to absorb them to the urban context.

The present article is analyzed the subjective perception of the neighborhood's residents especially the poor by a brief introduction of the geographical approach to the quality of life. The case study of this article was Islamabad neighborhood in Zanjan

As this paper focuses on the participation assessment of quality of life in these neighborhoods, also has seen a collaborative approach under the power of the government as a solutions. The findings of the questionnaire (that there is no scope for detailed discussion here) have shown that the relationship between the neighborhoods, willing to cooperate and collaborate in order to resolve the problems in this area is very high. Therefore the best way of intervention is using cooperative and synergistic approaches between people and different Management institutions. Given the foregoing, it must be said that there is the Partnership (now potentially) in the neighborhood. 
Why this potential is not turned to de facto yet have to be known in the dominant management structures of society, centralized policy-oriented management, the nonrealization of civil rights, dependent of the people to the government, lack of the facilitator and navigator, lack of attention to cooperative approaches of management, lack of supply structures and social welfare, distributive injustice in subsidies and services to different areas and.... But altogether, it must be said that, what can be effective in enhancing the quality of life in these neighborhoods are not only the charity policies that will lead to more dependence, but also the empowerment approach or the so-called teaching the fishing that can be effective in power increasing and consequently the quality of life. Of course this does not mean that the distribution policy of the managements, government's supply and social welfare (social policy) have to be forgotten, but the intent is the synergy of nation and government to solve the problems and improve the quality of life firmly integrated and stable.

\section{References}

Afrough, Emad (1377)," space and social inequality present a model for the spatial segregation and its consequences", Tehran, Tarbiat Modarres University.

Tavakoli, M. (1384), "identifying the criminality centers in Zanjan city by using geographic information system GIS", Ma thesis in the field of command and security management, law graduate thesis, University of police (officer) Tehran, Officer University

Smith, David (1381), "Quality of life: human welfare and social justice" translate by Hussein Hataminezhad and Hekmat shahi Ardebili, The political - economic information, seventeenth year, No. 185 and 186.

Haji-Yousefi, Ali (1381), 'quality of life and basic strategies", Journal of Work, No. 8, Tehran, University of Social Welfare and rehabilitation.

Kharazmi, Shahindokht (1382), "Quality of life and happiness index", Journal of Tadbir, No. 20, Tehran.
Zebardast, Esfandiyar. (1380), "The use of "AHP" in urban and regional planning", Fine Arts Magazine, No. 10, Tehran, Faculty of Fine Arts of Tehran University.

Seyyed Mirzaee, Mohammad (1376), "population and quality of life", Journal of Population, No. 21 and 22, Tehran, NOCR.

Sarafi, Mozafar (1381), "improving the quality of life", journal of the municipalities, No. 27, Tehran, country's municipal and rural organizations.

Sarafi, Mozafar (1383), "the problem called informal settlements, the solution called empowerment", journal of the municipality, No. 66, Tehran, country's municipal and rural organizations.

Kokabee Afshin, Pourjafar MR, taghavi, Aliakbar (1384), "planning the quality of life in urban centers, indicators and definitions", Journal of Urban query, No. 12, Tehran.

Armanshahr Consulting Engineers, (1383), Detailed plan of Zanjan.

Armanshahr Consulting Engineers, (1386), Revised detailed plan of Zanjan.

Harvey, David (1376), "city and social justice", translated by Farukh Hesamiyan ,M.Reza Haeri and Behroz Monadi zadeh, first winter publish, Processing and urban planning firms.

Phillips, David, Quality of life concept, policy and practice, London, Rutledge 2006, p. p. 276

Harvey, David (1996): Justice .Nature \& Geography of difference, Blackwell publishers, Oxford, UK.

Daphne Greenwood, local indicator quality of life, Washington DC, 2001 . 